\title{
Continuum Wannier-Stark Ladders Strongly Coupled by Zener Resonances in Semiconductor Superlattices
}

\author{
M. Helm, ${ }^{1}$ W. Hilber, ${ }^{1}$ G. Strasser, ${ }^{2}$ R. De Meester, ${ }^{3}$ F. M. Peeters,${ }^{3}$ and A. Wacker ${ }^{4}$ \\ ${ }^{1}$ Institut für Halbleiter- und Festkörperphysik, Universität Linz, A-4040 Linz, Austria \\ ${ }^{2}$ Institut für Festkörperelektronik, Technische Universität Wien, A-1040 Wien, Austria \\ ${ }^{3}$ Department of Physics, University of Antwerp (UIA), B-2610 Wilrijk, Belgium \\ ${ }^{4}$ Institut für Theoretische Physik, Technische Universität Berlin, D-10623 Berlin, Germany
}

(Received 9 November 1998)

\begin{abstract}
We report the first experiment which relates electronic transport and intraband optical absorption in a biased superlattice. Wannier-Stark ladders far in the continuum and strongly coupled by Zener resonances are observed in an $n$-type GaAs/AlGaAs superlattice using infrared spectroscopy. Additional transport measurements reveal the formation of electric-field domains, with electrons tunneling resonantly to the next-nearest superlattice period. [S0031-9007(99)08911-5]
\end{abstract}

PACS numbers: 73.20.Dx, 73.40.Gk, 78.30.Fs, 78.66.Fd

The physics of semiconductor superlattices [1] has been the subject of intense research for almost three decades, in particular, in relation to phenomena such as Bloch oscillations and Wannier-Stark ladders. If an electric field is applied perpendicular to the layers of a periodic superlattice, the energy bands ("minibands") are known to split up into a ladder of localized states, the Wannier-Stark ladder (WSL). Its existence has been demonstrated through optical experiments, involving transitions from the valence to the conduction band [2]. In a semiclassical picture, the electron can reach the inflection point and the edge of the mini-Brillouin zone, giving rise to negative differential resistance (NDR) in the current-voltage characteristics and to Bloch oscillations $[3,4]$, which is the semiclassical analog of the Wannier-Stark ladder. Bloch oscillations have been observed directly in the time domain by optically exciting electron wave packets with a subpicosecond laser pulse [5]. The NDR, however, also arises in more weakly coupled superlattices, where transport proceeds by sequential resonant tunneling, and is usually accompanied by the formation of electric-field domains [6,7]. In the very high-field regime, the resonant Zener breakdown has been observed [8] as well as infrared emission from Wannier-Stark ladders [9]. Intersubband absorption experiments under application of an electric field have only been performed on isolated quantum wells.

A crucial issue for the understanding of any semiconductor structure is the question of how the current-voltage characteristics are related to the energy level structure. For superlattices this is particularly important, if one wishes to learn about the relation between NDR and Wannier-Starkladder formation. Guided by this question, we simultaneously investigate the transport and the intraband optical properties of a semiconductor superlattice [10] in a vertical electric field, which exhibits NDR, domain formation, and the formation of Wannier-Stark ladders in the continuum. By employing an $n$-type SL, we deal exclusively with electron states, and we spectroscopically study inter-Wanner-
Stark-ladder transitions within the conduction band. This paper represents the first ever infrared (IR) absorption measurement under high-field, high-current vertical-transport conditions in a semiconductor superlattice. We observe tunneling to the next-nearest quantum well, and direct as well as indirect Wannier-Stark-ladder transitions into continuum states, which are strongly coupled by Zener resonances $[8,11]$.

The SL studied consists of 300 periods of GaAs quantum wells (QW, $50 \AA$ wide) and $\mathrm{Al}_{0.29} \mathrm{Ga}_{0.71} \mathrm{As}$ barriers (80 $\AA$ wide), sandwiched between $n^{+}$GaAs contact layers and grown on a semi-insulating substrate by molecular beam epitaxy. The parameters have been chosen in a way to obtain a rather narrow first miniband (calculated width $\Delta_{1}=1.2 \mathrm{meV}$ ), but relatively wide excited minibands (width of second miniband $\Delta_{2}=$ $30 \mathrm{meV}$ ), which are located in the continuum above the barriers (see inset of Fig. 1). Whereas the latter condition

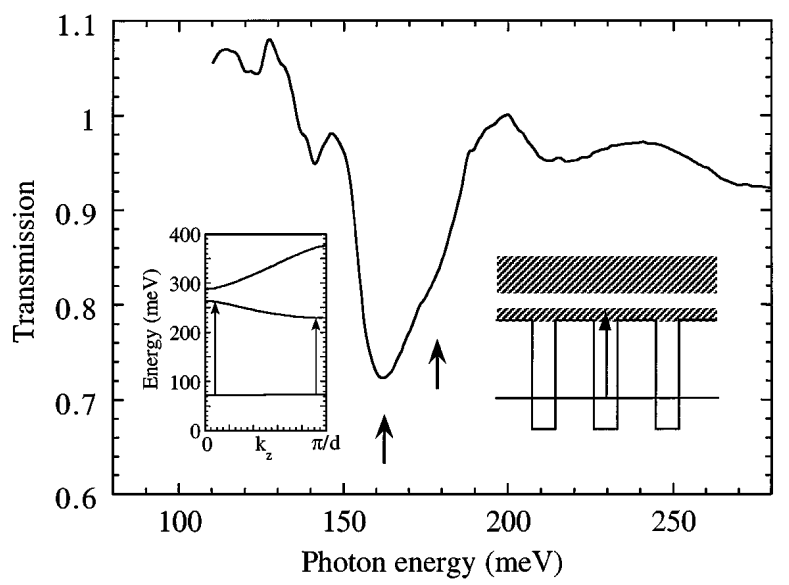

FIG. 1. Interminiband transmission spectrum for zero electric field. The insets show the absorption process in $k$ space and real space, respectively. The main absorption and the highenergy shoulder, due to the singularities in the joint-density of states, are indicated by arrows. 
implies strong coupling between adjacent quantum wells via the excited states, the former condition helps to avoid excessive current densities, usually encountered in strongly coupled SLs. Such high-current densities $(j \approx$ $10^{3}$ to $10^{4} \mathrm{~A} / \mathrm{cm}^{2}$ ) would be unacceptable for the IR absorption experiments, where mesa devices of the order of $1 \mathrm{~mm}^{2}$ area are required. The SL is doped in the center $30 \AA$ of the barriers, giving an areal electron concentration of $2.25 \times 10^{11} \mathrm{~cm}^{-2}$ per period (this results in a Fermi energy of $E_{F}=8 \mathrm{meV}$ at low temperature and when impurity effects [12] are neglected). This relatively high doping is introduced to provide enough carriers for the absorption measurements. Standard lithographic and etching techniques were used to fabricate mesas with sizes between $100 \times 100 \mu \mathrm{m}^{2}$ and $1 \times 1 \mathrm{~mm}^{2}$. For the IR absorption measurements, performed with a Bruker IFS66 Fourier transform spectrometer, the samples with the larger mesa structures were prepared with wedged facets in order to couple the IR light to the intersubband transitions via total internal reflection. The IR beam in the spectrometer was focused to a spot size of $<1 \mathrm{~mm}$ with a condensing unit. For the measurements with electric field, the spectrometer was operated in a gated step-scan mode, synchronized with the electric-field pulses [13]. All measurements were performed at $T=10 \mathrm{~K}$.

Figure 1 shows the zero-field interminiband transmission spectrum of the superlattice, obtained by ratioing the $p$-polarized (active) and the $s$-polarized (inactive) transmission signal. The main absorption maximum at $164 \mathrm{meV}$ and the high-energy shoulder at $180 \mathrm{meV}$ (both indicated by arrows in Fig. 1) are due to the well-known singularities of the joint density of states at $k_{z}=\pi / d$ and $k_{z}=0$, respectively, where $k_{z}$ is the wave vector along the growth direction and $d$ is the superlattice period [14]. This is illustrated in the left inset, which shows the three lowest minibands. (A calculated zero-field interminiband absorption spectrum is shown below in the lowest curve of Fig. 4).

In Fig. 2 the current-voltage characteristic of a $200 \mu \mathrm{m}$ mesa is shown. At low bias the conduction is Ohmic and proceeds by ground-state-to-ground-state tunneling up to a voltage of $0.5 \mathrm{~V}$ [15]. There the SL breaks up into a low- and a high-field domain; in the latter the electrons tunnel from the ground state in one well to an excited state in a subsequent well (usually the adjacent one). Its extent increases with increasing bias, until the field distribution is again homogeneous and the current rises steeply. In the present superlattice this occurs at $27 \mathrm{~V}$, i.e., the voltage drop per period is $27 \mathrm{~V} / 300=90 \mathrm{mV}$. Since this is only about half the value of the subband separation, $E_{2}-E_{1} \approx 170 \mathrm{meV}$, we conclude that in the high-field domain the ground state is approximately aligned with the first excited state of the next-nearest well, and electrons directly tunnel to the next-nearest SL period, traversing two barriers and one well (see inset of Fig. 2). This is the first observation, to our knowledge, of resonant tunneling over two periods in a regular superlattice [16]. At present,

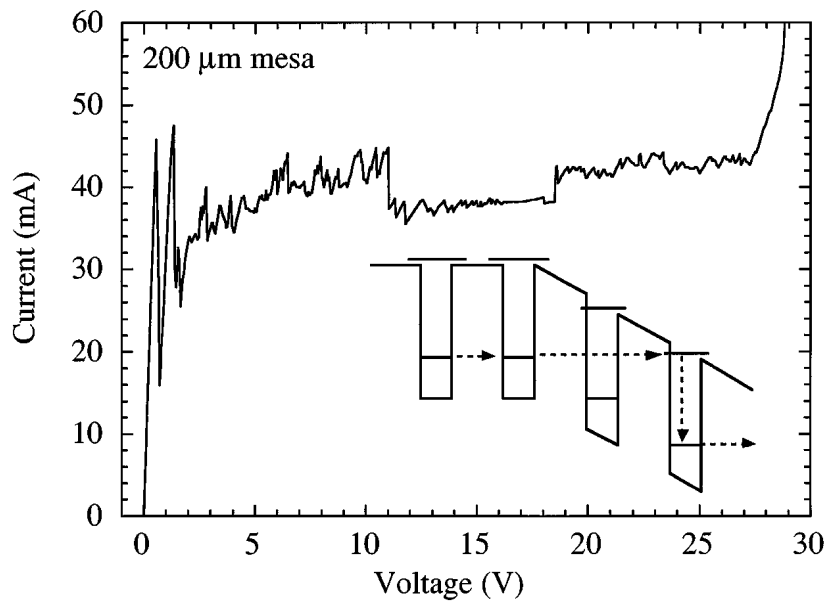

FIG. 2. Current-voltage characteristic of the superlattice. The inset shows the conduction-band edge near the boundary of the low- and high-field domain together with a schematic of the transport process.

there is no quantitative theory for this phenomenon; it would require consideration of additional transition processes via intermediate states.

For the measurement of the IR absorption spectrum in a vertical electric field, voltage pulses of about 5-10 $\mu \mathrm{s}$ duration with a repetition rate of a few hundred $\mathrm{Hz}$ were applied to a $1 \times 1 \mathrm{~mm}^{2}$ superlattice mesa. The transmission change, $\Delta T=T(F) / T_{0}$, is measured by dividing the signal during the electric-field pulse, $T(F)$, by the signal taken a few ten $\mu$ s after the pulse, $T_{0}$ [13]. Figure 3 shows a series of traces for increasing voltage. Note that minima in the signal correspond to decreased

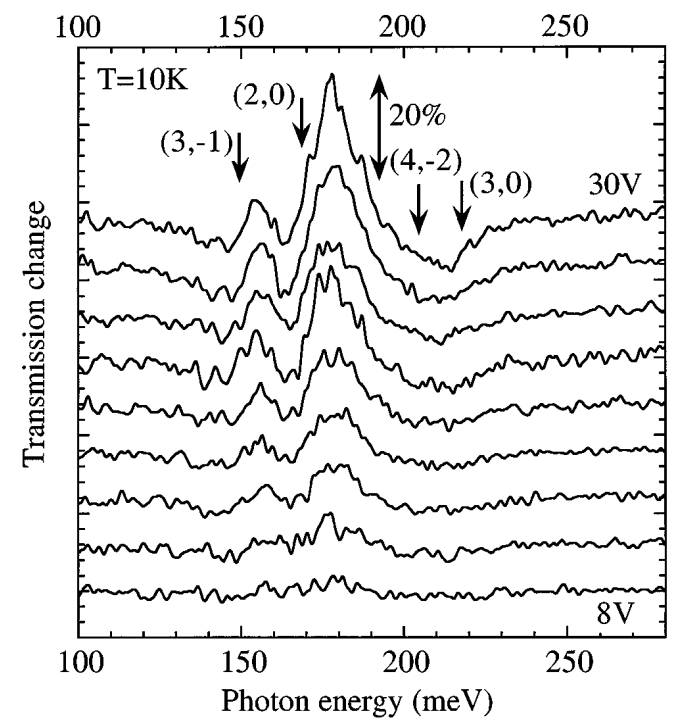

FIG. 3. Differential transmission spectrum $\left(T(F) / T_{0}\right)$ of the superlattice for a series of bias voltages between 8 and $30 \mathrm{~V}$. Minima essentially correspond to electric-field-induced absorption lines. The arrows with the labels correspond to calculated transitions, which are explained in the text. 
transmission, i.e., they essentially correspond to absorption lines induced by the electric field. Remarkably, the positions of the maxima and minima hardly change with bias, but $\Delta T$ continuously increases, reaching up to $30 \%$ at the highest voltage. This is completely consistent with the observations in the $I-V$ characteristics. Two different electric-field values exist in the sample, which are realized in two spatial domains; one is close to zero (in the low-field domain) and the other one (in the high-field domain) is approximately $F=\left(E_{21} / 2\right) / e d=$ $85 \mathrm{mV} / 130 \AA=65 \mathrm{kV} / \mathrm{cm}$, corresponding to tunneling to the next-nearest SL period. Increasing the voltage only increases the relative length of the high-electric-field domain, resulting in a larger optical signal. Above $30 \mathrm{~V}$, the current increases so steeply that no measurements can be performed in this regime.

Understanding the transmission change spectra of Fig. 3 requires a calculation of the absorption coefficient $\alpha$ under bias. For this purpose we have calculated the energy spectrum, the wave functions, and the optical matrix elements between the ground state of one period and all excited states, using a finite system of $N=19 \mathrm{SL}$ periods. The absorption coefficient $\alpha$ is proportional to

$$
\alpha_{n}(\omega)=\text { const } \times \sum_{n^{\prime}=1}^{N} f_{n n^{\prime}} \frac{\Gamma / \pi}{\left(E_{n^{\prime}}-E_{n}-\hbar \omega\right)^{2}+\Gamma^{2}},
$$

where $n$ indicates the initial state (ground state of one SL period), $n^{\prime}$ are the final states, and $f_{n n^{\prime}}$ is the oscillator strength, obtained from the optical matrix elements. A Lorentzian broadening $\Gamma=7 \mathrm{meV}$ of each transition (chosen to best reproduce the experiment) is assumed. The calculated absorption coefficient is plotted in Fig. 4 for electric fields from $F=0$ up to $F=70 \mathrm{kV} / \mathrm{cm}$. Only up to $20 \mathrm{kV} / \mathrm{cm}$ the splitting of the interminiband absorption into a regular Wannier-Stark ladder can be observed. At higher fields, strong mixing of several continuum Wannier-Stark ladders occurs due to Zener coupling and the interpretation of the spectra is far from straightforward. The strong Zener coupling results from the fact that $e F d$ is much larger than the minigaps in the continuum.

For direct comparison with experiment, we calculate $\alpha(0)-\alpha(F)$, which is proportional to $T(F) / T_{0}$ for small absorption changes. This is plotted in the top part of Fig. 4 for $F=50,60$, and $70 \mathrm{kV} / \mathrm{cm}$. Comparison with the experimental traces (Fig. 3) shows that most of the experimental features can be reasonably well reproduced for a field of $F=60 \mathrm{kV} / \mathrm{cm}$ (drawn by thicker lines for clarity); only the maximum at $\approx 180 \mathrm{meV}$ is larger than predicted, probably since its strength is very sensitive to the exact shape of the shoulder in the $T_{0}$ [or $\alpha(0)$ ] spectrum. The above field corresponds to a voltage drop of $80 \mathrm{meV}$ per SL period, which is very close to half the energy separation $E_{21}$, and thus confirms the tunneling to the next-nearest SL period. The calculated positions are also indicated in Fig. 3 by arrows. The relative positions

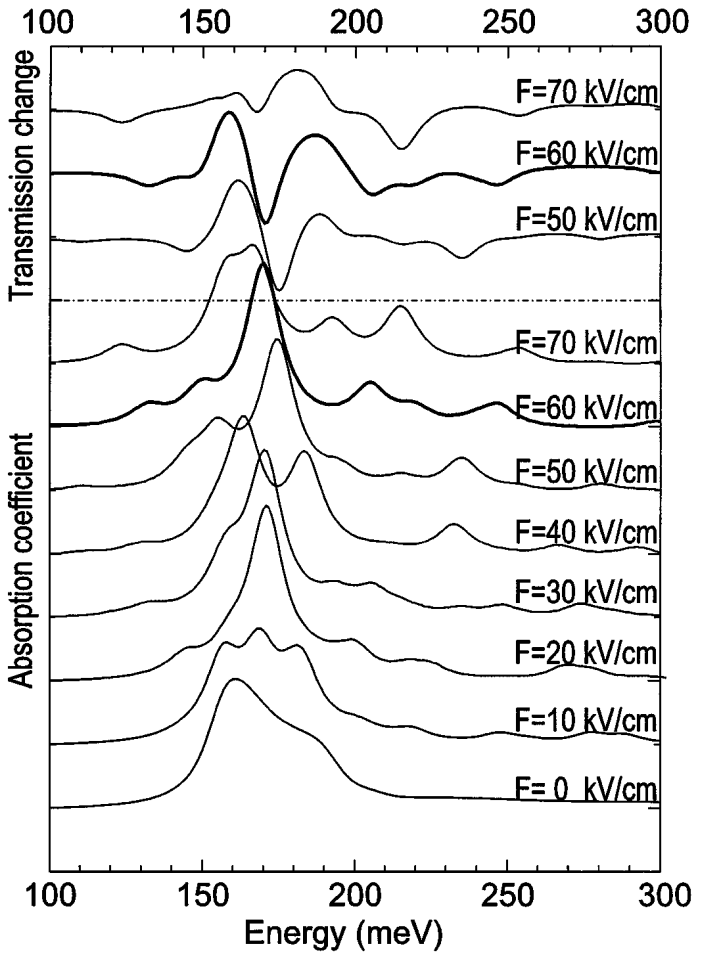

FIG. 4. Absorption coefficient calculated for electric fields from 0 to $70 \mathrm{kV} / \mathrm{cm}$. In addition, the transmission change is shown for 50,60 , and $70 \mathrm{kV} / \mathrm{cm}$ in the upper panel to facilitate comparison with the experiment (see text). The experimentally relevant $60 \mathrm{kV} / \mathrm{cm}$ spectra are plotted with thicker lines (compare with Fig. 3.

agree very well, even the absolute values apart from a rigid shift of about $5 \mathrm{meV}$.

In order to obtain a more graphical understanding of the transitions dominating the absorption spectrum, we attempt a classification of the energy levels in terms of pure WSL quantum numbers, neglecting the interaction due to Zener coupling for the moment. The energy spectrum can be written as $E_{m, p}=\varepsilon_{m}+p e F d$, where $m=1,2, \ldots$ is the (zero-field) miniband index and $p=$ $\ldots-1,0,1, \ldots$ is the spatial WSL index [17]. Such an (approximate) assignment can be done by inspecting in detail the wave functions and oscillator strengths over several SL periods. Transitions occur from states $|m=1, p\rangle$ to $\left|m^{\prime}, p^{\prime}\right\rangle$ and, for brevity, we term such a transition $\left(m^{\prime}, p^{\prime}-p\right)$. Figure 5 shows the resulting assignment graphically. The main part of the relevant wave functions are drawn bold and also the calculated absorption coefficient is shown for comparison. The main observed transitions (indicated by arrows) are, from low to high energies, $(3,-1),(2,0)$ (this is the main vertical intersubband transition), $(4,-2)$, and $(3,0)$ (compare also labels in Fig. 3). The latter two are strongly mixed, since they are close to a Zener anticrossing. Besides the direct transitions, we thus observe indirect transitions up to the next-nearest downhill $\mathrm{QW}\left(p^{\prime}-p=-2\right)$, and up to 


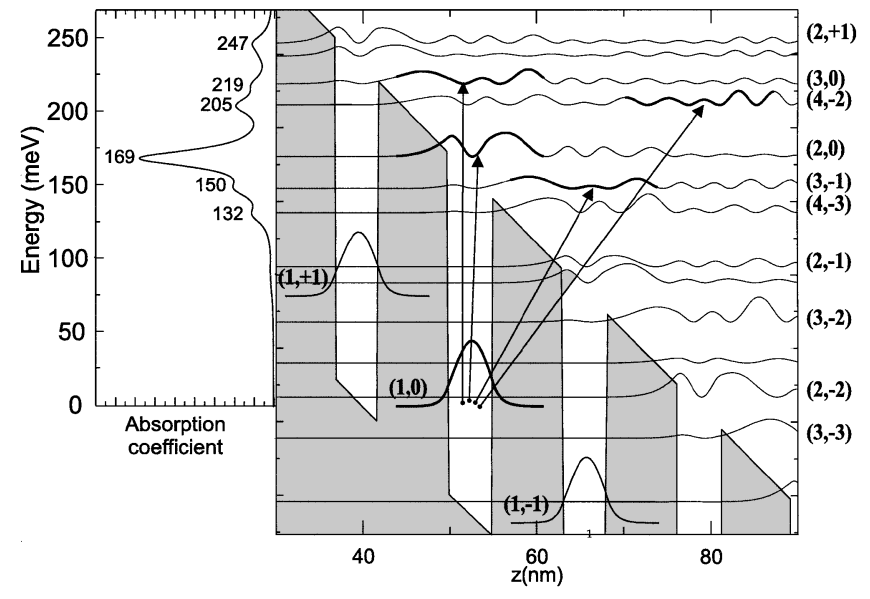

FIG. 5. Conduction-band profile, energy levels, and squared wave functions drawn for four superlattice periods with an electric field of $60 \mathrm{kV} / \mathrm{cm}$. The experimentally observed transitions are indicated by arrows and the main parts of the respective wave functions are emphasized by thicker lines. The numbers on the right represent an (approximate) assignment in terms of WS states (see text). In addition, the calculated absorption coefficient is shown on the left for comparison, including the peak photon energies.

states related to the fourth miniband $\left(m^{\prime}=4\right)$. Note that the $(4,-2)$ state is as far as $200 \mathrm{meV}$ above the top of the barrier, when measured at its associated quantum well, $p^{\prime}-p=-2$. The calculated lowest- and highestenergy transitions (at 132 and $247 \mathrm{meV}$ ) predicted by the calculation are not resolved in the experiment.

As seen in Fig. 5, the continuum WSL wave functions are distinctly different from the usual, symmetrically localized, tight-binding type wave functions [17]. They are very asymmetric and extend far towards the anode, partly exhibiting Airy-function character. Note also that, due to the finiteness of the structure used in the calculation, no perfect periodicity of the solution for the wave functions under a translation $z \rightarrow z+d$ and $E \rightarrow E-e F d$ is obtained. The absorption coefficient, which is the $o b$ servable quantity, fulfills this symmetry to a much better degree, when calculated with a finite broadening $\Gamma$. In other words, the oscillator strength per finite energy interval $\Gamma$ is independent of the quantum number $p$, despite differences in the wave functions. This shows the relation of $\Gamma$ to the coherence length; when $\Gamma$ is very small, the wave functions more easily feel the edges of the finite SL [18].

In summary, we have evidenced the existence of interpenetrating, Zener-coupled Wannier-Stark ladders in the continuum of a superlattice, as far as $200 \mathrm{meV}$ above the top of the barriers. The Wannier-Stark ladders are formed under conditions of negative differential resistance and domain formation, where in the high-field domain transport proceeds by resonant tunneling to the nextnearest superlattice period.

We are very grateful to K. Unterrainer and G. Bauer for helpful discussions. This work was supported by the FWF under Project No. P10211, by the "Jubiläumsfonds der OeNB" under project No. 5987, by the GMe (all in Austria), and by the FWO-VI (Belgium). R.D.M. is supported by IWT (Belgium).

[1] Semiconductor Superlattices, edited by H.T. Grahn (World Scientific, Singapore, 1995).

[2] E. E. Mendez, F. Agullo-Rueda, and J. M. Hong, Phys. Rev. Lett. 60, 2426 (1988); P. Voisin et al., Phys. Rev. Lett. 61, 1639 (1988).

[3] L. Esaki and R. Tsu, IBM J. Res. Dev. 14, 61 (1970).

[4] A. Sibille et al., Superlattices Microstruct. 13, 247 (1993).

[5] K. Leo, Semicond. Sci. Technol. 13, 249 (1998), and references therein.

[6] H. T. Grahn, H. Schneider, and K. von Klitzing, Phys. Rev. B 41, 2890 (1990); S. H. Kwok et al., Phys. Rev. B 51, 9943 (1995).

[7] A. Wacker, in Theory of Transport Properties of Semiconductor Nanostructures, edited by E. Schöll (Chapman and Hall, London, 1998), p. 321.

[8] A. Sibille, J. F. Palmier, and F. Laruelle, Phys. Rev. Lett. 80, 4506 (1998).

[9] G. Scamarcio et al., Phys. Rev. B 57, R6811 (1998).

[10] Transport and interband optical experiments on the same sample have been reported in A. Sibille, J. F. Palmier, and F. Mollot, Appl. Phys. Lett. 60, 457 (1992).

[11] H. Schneider, H. T. Grahn, K. v. Klitzing, and K. Ploog, Phys. Rev. Lett. 65, 2720 (1990).

[12] A. Wacker, A.-P. Jauho, S. Zeuner, and S. J. Allen, Phys. Rev. B 56, 13268 (1997).

[13] W. Hilber, M. Helm, K. Alavi, and R. N. Pathak, Appl. Phys. Lett. 69, 2528 (1996).

[14] M. Helm et al., Phys. Rev. B 48, 1601 (1993); M. Helm, Semicond. Sci. Technol. 10, 557 (1995).

[15] Since in our sample the broadening is larger than the width of the first miniband, we are rather in the regime of sequential resonant tunneling than of miniband conduction. See also A. Wacker and A.-P. Jauho, Phys. Rev. Lett. 80, 369 (1998).

[16] Note that tunneling over several periods has been recently observed in superlattices with a basis by A. Kristensen, P. E. Lindelof, C. B. Sørensen, and A. Wacker [Semicond. Sci. Technol. 13, 910 (1998)].

[17] J. Bleuse, G. Bastard, and P. Voisin, Phys. Rev. Lett. 60, 220 (1988); M. Saitoh, J. Phys. C 5, 914 (1972).

[18] C. Rauch et al., Phys. Rev. Lett. 81, 3495 (1998). 